\title{
Cell-veto Monte Carlo algorithm for long-range systems
}

\author{
Sebastian C. Kapfer ${ }^{1, *}$ and Werner Krauth ${ }^{2}$,† \\ ${ }^{1}$ Theoretische Physik 1, FAU Erlangen-Nürnberg, Staudtstr. 7, 91058 Erlangen, Germany \\ ${ }^{2}$ Laboratoire de Physique Statistique, Ecole Normale Supérieure / PSL Research University, \\ UPMC, Université Paris Diderot, CNRS, 24 rue Lhomond, 75005 Paris, France
}

(Dated: August 14, 2021)

\begin{abstract}
We present a rigorous efficient event-chain Monte Carlo algorithm for long-range interacting particle systems. Using a cell-veto scheme within the factorized Metropolis algorithm, we compute each single-particle move with a fixed number of operations. For slowly decaying potentials such as Coulomb interactions, screening line charges allow us to take into account periodic boundary conditions. We discuss the performance of the cell-veto Monte Carlo algorithm for general inverse-powerlaw potentials, and illustrate how it provides a new outlook on one of the prominent bottlenecks in large-scale atomistic Monte Carlo simulations.
\end{abstract}

Markov-chain Monte Carlo is one of the most widely used computational methods in the natural sciences. It samples a high-dimensional space of configurations $c$ according to a probability distribution $\pi(c)$. In the physical sciences, $\pi$ generally corresponds to the Boltzmann distribution $\pi(c)=\exp [-\beta E(c)]$, where $\beta$ is the inverse temperature and $E$ the system energy. The core of most Monte Carlo computations is the Metropolis algorithm [1, which accepts a trial move from configuration $i$ to configuration $f$ with probability

$$
p^{\operatorname{Met}}(i \rightarrow f)=\min \{1, \exp [-\beta(E(f)-E(i))]\} .
$$

The acceptance probability Eq. 1 satisfies the detailed balance condition, $\pi(i) p^{\operatorname{Met}}(i \rightarrow f)=\pi(f) p^{\operatorname{Met}}(f \rightarrow i)$, that leads to exponential convergence towards the stationary distribution $\pi(c)$, if ergodicity is assured [2]. Moving from one configuration to another requires evaluating the induced change of the system energy. In most classical $N$-particle simulations, the system energy is a sum over pair terms: $E=\sum_{\langle k, l\rangle} U_{k l}=\sum_{\langle k, l\rangle} U\left(\mathbf{r}_{k l}\right)$ with the pair potential $U$ and the interparticle distances $\mathbf{r}_{k l}=\mathbf{r}_{l}-\mathbf{r}_{k}$. The evaluation of the system energy generally takes $\mathcal{O}\left(N^{2}\right)$ operations, and the computation of the energy change upon moving a single particle takes $\mathcal{O}(N)$ operations. For a potential with finite support, the change of the system energy for moving one particle is computed in $\mathcal{O}(1)$. To speed up the evaluation, potentials with infinite support, such as the LennardJones and other moderately long-ranged potentials, are truncated beyond an effective interaction range. This approximation is however known to alter the equilibrium properties 3, 4. Strongly long-ranged potentials, as they appear in electrostatics and gravity, do not allow for the definition of a finite interaction range and require specialized techniques for determining the system energy to high precision. Ewald summation [5, 6], for example, adds and subtracts smooth charge distributions localized around the point particles. With periodic boundary conditions, this turns the long-ranged part of the interaction into a rapidly converging sum in Fourier space. Ewald summation computes the system energy in $\mathcal{O}\left(N^{3 / 2}\right)$, tak- ing into account periodically replicated images of the particles [6, 7]. Its refinements further reduce the burden of the system-energy computation by discretizing the charge density [8] or by exploiting large-scale uniformity 9]. Still, in many outstanding applications in the natural sciences, the evaluation of long-ranged potentials remains a computational bottleneck. Implementing Ewald summation is particularly difficult if periodic boundary conditions are not realized in all dimensions, as for example in slab geometries [10, 11.

In this paper, we present a rigorous Monte Carlo algorithm for $N V T$ particle systems with long-ranged interactions that does not evaluate the system energy, in contrast to virtually all existing Markov-chain Monte Carlo algorithms [2. This change of perspective opens up many opportunities: Based on a cell-veto scheme within the factorized Metropolis algorithm [12, it implements a single-particle move in complexity $\mathcal{O}(1)$ without any truncation error. For moderately long-ranged potentials, such as Lennard-Jones or dipolar interactions, the step size is independent of the system size, and the algorithm is effectively constant-time. For strongly long-ranged interactions, as the Coulomb forces, the single-move step size slightly decreases with $N$. For concreteness, we will consider a fixed hypercubic box of size $L^{D}$ with periodic boundary conditions, where $D$ is the dimension of physical space. The generalization to slab geometries is straightforward.

In contrast to the Metropolis algorithm of Eq. 1, the pairwise factorized algorithm 12 accepts moves with the probability

$$
p^{\mathrm{fact}}(i \rightarrow f)=\prod_{\langle k, l\rangle} \underbrace{\min \left\{1, \exp \left[-\beta \Delta U_{k l}(i \rightarrow f)\right]\right\}}_{p_{k l}(i \rightarrow f)},
$$

where $\Delta U_{k l}$ is the change in the pair potential between particles $k$ and $l$. In our algorithm, we never explicitly evaluate the function $p^{\text {fact }}$. Rather, the product of probabilities on the rhs of Eq. (2) is interpreted as a condition that is true if all its factors are true. The move $i \rightarrow f$ is thus accepted by consensus, namely if each pair $\langle k, l\rangle$ 

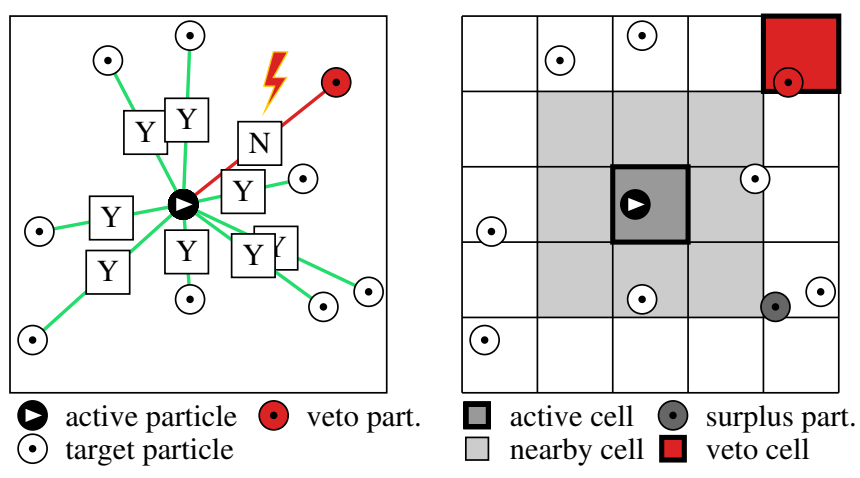

FIG. 1. (Color online) Pairwise factorized Monte Carlo algorithm. Left: The move of the active particle is vetoed by one target particle, so that the necessary consensus (all "Y") is not reached (see Eq. (2)). Right: In the cell-veto algorithm, vetos are provisionally solicited on the cell level (between the active cell $\mathcal{C}_{a}$ and the target cell $\mathcal{C}_{t}$ ) before being confirmed for the active particle, at $\mathbf{r}_{a} \in \mathcal{C}_{a}$ and the target particle, at $\mathbf{r}_{t} \in \mathcal{C}_{t}$. Nearby and surplus particles are treated differently.

independently accepts the move with probability $p_{k l}[12$ (see Fig. 1). Instead of computing the energy to high precision, we will compute upper bounds for the veto probability $1-p_{k l}$ by embedding particles $k$ and $l$ into cells $\mathcal{C}_{k}$ and $\mathcal{C}_{l}$, respectively. To identify particles vetoing the move, one rapidly identifies cell vetos and inspects the contents of corresponding cells to determine whether the cell vetos are confirmed on the particle level (see Fig. 1).

In continuum space, two configurations $i$ and $f$ with $i \neq f$ can be infinitesimally close to each other. For regular potentials, this implies that the change of pair energies $\Delta U_{k l}(i \rightarrow f)$, and therefore the veto probability $1-p_{k l}$, are infinitesimal as well. In the event-chain algorithm [12, 13, a proposed move $i \rightarrow f$ consists in the infinitesimal displacement of an "active" particle $a$ in a direction $\hat{\mathbf{e}}$ : The proposed move is $\mathbf{r}_{a}(i) \rightarrow \mathbf{r}_{a}(f)=$ $\mathbf{r}_{a}(i)+\hat{\mathbf{e}} \mathrm{d} s$, where $\mathrm{d} s$ is an infinitesimal time increment. The active particle keeps moving in the same direction until a move is finally vetoed by a target particle $t$. The target particle then becomes the new active particle, i. e., the proposed move is $(i, a, \hat{\mathbf{e}}) \rightarrow(f, a, \hat{\mathbf{e}})$, and if vetoed by particle pair $\langle a, t\rangle$, the configuration is changed to $(i, t, \hat{\mathbf{e}})$. This implements a "lifted" Markov chain 14 with two additional variables $a$ and $\hat{\mathbf{e}}$, which trivially projects to the physical space with the proper Boltzmann distribution. Veto probabilities $1-p_{a t}$ are infinitesimal. Two simultaneous vetos are thus prevented from arising from different target particles. Detailed balance is violated (the reverse move $\mathbf{r}_{a}(f)=\mathbf{r}_{a}(i)-\hat{\mathbf{e}} \mathrm{d} s$ is never proposed). However, the event-chain algorithm satisfies the global-balance condition

$$
\sum_{i} \pi(i) p(i \rightarrow f)=\pi(f)
$$

sufficient for exponential convergence to the equilibrium distribution on the accessible configurations. To ensure ergodicity, both the active particle and the direction of motion are periodically reset to random values (see Supp. 2). Lifted Markov chains have been shown to improve convergence speed in many cases, and also to lower the dynamical critical scaling exponents [14 17].

The core of an event-chain program consists in determining the step size $\Delta s$ to the next particle event and in identifying the vetoing target particle $t$, rather than explicitly programming small time increments (see Fig. 22 a). The actual move then merely consists in updating the active particle position as $\mathbf{r}_{a} \rightarrow \mathbf{r}_{a}+\hat{\mathbf{e}} \Delta s$ and in changing the active particle to $t$. For long-ranged potentials, $t$ can be far away from the active particle. At any instant during the simulation, the veto probability of a potential target particle $t$ is given by the particle-event rate $q$, defined via a directional derivative of the pair potential,

$$
1-p_{a t}=q\left(\mathbf{r}_{a t}\right) \mathrm{d} s=\beta\left[-\hat{\mathbf{e}} \cdot \nabla U_{a t}\right]^{+} \mathrm{d} s
$$

with $[\cdot]^{+}=\max (0, \cdot)$. For long-ranged potentials, $q$ carries over large distances (see Fig. 2b, c). Particleevent distances $r_{a t}$ are distributed as $q\left(r_{a t}\right) g\left(r_{a t}\right)$, where $g$ is the radial distribution function, and thus exhibit the same long-ranged tail. In contrast, the displacement between events, i. e., the step size $\Delta s$, decays exponentially within a few interparticle distances, see Fig. 2k. For each pair $\langle a, t\rangle$, the event time $\Delta s_{t}$ can be computed in $\mathcal{O}(1)$, so that the event-chain algorithm can be implemented in $\mathcal{O}(N)$ per particle event [18, by iterating over all target particles. The earliest veto will define the step size $\Delta s$ and the active particle for the next step.

For a homogeneous system (with a bounded particle density), the complexity per particle event can be re-

a)

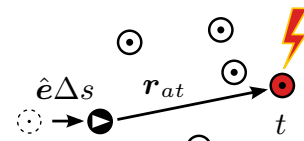

b)
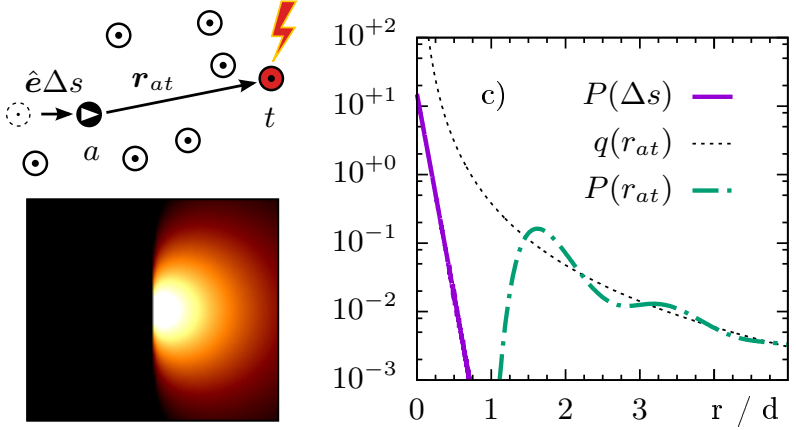

FIG. 2. (Color online) Event-chain algorithm for a longranged dipolar potential in two dimensions, $\beta U=10 \times(d / r)^{3}$. a) The active particle takes infinitesimal moves in the $+x$ direction. At time $\Delta s$, a move is vetoed by particle $t$, at event-time distance $\mathbf{r}_{a t}$. The vetoing particle $t$ becomes the new active particle and starts to move in the $+x$ direction. $b$ ) Heatmap representation of the particle-event rate $q\left(\mathbf{r}_{a t}\right)$. The active particle is in the center, black corresponds to $q=0$. c) Probability distributions of the step size $\Delta s$ taken by the active particle and of particle-event distances $r_{a t}$. 
duced from $\mathcal{O}(N)$ to $\mathcal{O}(1)$ by establishing upper bounds for the particle-event rate which hold irrespective of the precise particle positions. Concretely, we superimpose a fixed regular grid onto the system, with cells typically containing at most one particle (see Fig. 1, rare "surplus" particles are treated separately). The particle-event rate between the active particle in cell $\mathcal{C}_{a}$ and a target particle in cell $\mathcal{C}_{t}$ is bounded from above by the cell-veto rate

$$
Q\left(\mathcal{C}_{a}, \mathcal{C}_{t}\right)=\max _{\mathbf{r}_{a} \in \mathcal{C}_{a}, \mathbf{r}_{t} \in \mathcal{C}_{t}} q\left(\mathbf{r}_{t}-\mathbf{r}_{a}\right)
$$

This quantity depends only on the pair potential and the relative positions of the two cells and can be tabulated before sampling starts. The cell-veto rate remains finite except for a few nearby cells that contain the hard-core singularities. In the case of point particles, these must include any cells that share corners with $\mathcal{C}_{a}$ (see Fig. 1). For efficiency, "nearby" cells may comprise a larger portion of the short-range features of $U$.

Excluding nearby and surplus particles, the total particle-event rate is bounded from above by the total cell-veto rate

$$
Q_{\text {tot }}=\sum_{\mathcal{C}_{t}} Q\left(\mathcal{C}_{a}, \mathcal{C}_{t}\right)
$$

which remains a constant throughout the simulation. The next cell veto can then be sampled in $\mathcal{O}(1)$ : The time is distributed exponentially

$$
P(\Delta s)=Q_{\text {tot }} \exp \left(-Q_{\text {tot }} \Delta s\right),
$$

so that $\Delta s$ is given through the logarithm of a uniform random number ([2], see Supp. 2). The cell veto is triggered by the cell $\mathcal{C}_{t}$ with probability $\sim Q\left(\mathcal{C}_{a}, \mathcal{C}_{t}\right)$. The selection of the target cell from all the non-nearby cells can also be accomplished in constant time (see below). If the vetoing cell $\mathcal{C}_{t}$ contains a particle, at position $\mathbf{r}_{t}$, it is then chosen as the target particle for a particle event with probability $q\left(\mathbf{r}_{a}+\hat{\mathbf{e}} \Delta s, \mathbf{r}_{t}\right) / Q\left(\mathcal{C}_{a}, \mathcal{C}_{t}\right)$. This longrange particle event must be put into competition with events triggered by nearby or surplus particles, which are handled as in the short-range event-chain algorithm [12] (see also Supp. 2). The number of nearby particles is naturally bounded. The number of surplus particles may be kept as small as desired by adapting the cell size. In practice, we use cells that are sufficiently small so that surplus particles appear only exceptionally. Consequently, a cell veto can effectively be processed constant time, and the performance of the cell-veto algorithm depends on the rate of cell vetos $Q_{\text {tot }}$.

The total cell-veto rate $Q_{\text {tot }}$ depends on the range of the pair potential. For inverse-power-law interactions, $U(r) \sim 1 / r^{n}$, the event rate for a bare particle scales as $q \sim 1 / r^{n+1}$ 19. In an infinite system, the total cell-veto rate $Q_{\text {tot }} \sim \int \mathrm{d}^{D} r q$ is finite for moderately long-ranged potentials, i. e., for $n>D-1$. This class includes dipolar forces in $D=2$ and $D=3$, as well as the LennardJones potential. In this case, the cell-veto algorithm is of complexity $\mathcal{O}(1)$.
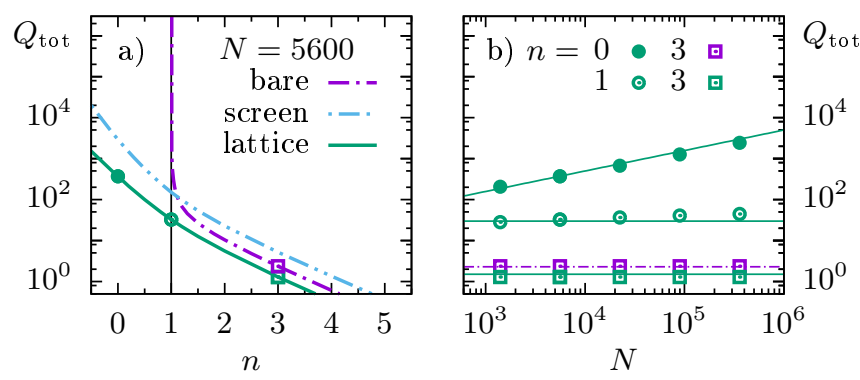

FIG. 3. (Color online) a) Total cell-veto rate $Q_{\text {tot }}$, Eq. 5. for inverse-power-law potentials in $D=2$. The event rate for bare particles diverges as $n \rightarrow D-1$ (vertical line) because of the presence of periodic images, while screened event rates stay finite. Solid bullets are the Coulomb system $(n=D-2$; for $2 \mathrm{D}, U(r) \sim-\ln r)$. b) Scaling of $Q_{\text {tot }}$ with system size $N$, for the screened-lattice algorithm (green solid), and for the bare-particle algorithm (purple dashed). The inclined line is $\sim N^{1 / 2}$.

For strongly long-ranged potentials $(n \leq D-1$, including Coulomb forces), the cell-veto rate in an infinite system diverges (see Fig. 3a). In the replicated-box representation of periodic boundary conditions (see Fig. 4), even the sum over all periodic images of a single target particle $(N=2)$ leads to an infinite particle-event rate. The sum may be regularized by adding uniformly charged line segments (parallel to the direction of motion ê) that neutralize each particle charge yet combined leave invariant the energy differences of the original system. Screening line charges can be defined for general potentials. For inverse-power-law interactions, the directional derivatives of the particle and line-charge potentials are

$$
\begin{aligned}
& \tilde{q}(\mathbf{r})=\beta \varepsilon d^{n} \times \frac{\hat{\mathbf{e}} \cdot \mathbf{r}}{r^{n+2}}, \\
& \tilde{l}(\mathbf{r})=\frac{\beta}{L} \times[U(\mathbf{r}+\hat{\mathbf{e}} L / 2)-U(\mathbf{r}-\hat{\mathbf{e}} L / 2)],
\end{aligned}
$$

where $\mathbf{r}$ is the folded-out distance vector between the active particle and a particular periodic image of the target particle. By vanishing monopole and dipole moments, $\tilde{q}+\tilde{l}$ asymptotically decays as $1 / r^{n+3}$, sufficient to render $Q_{\text {tot }}$ unconditionally convergent for Coulomb forces.

We may now define three distinct particle-event rates:

$$
q(\mathbf{r})= \begin{cases}{[\tilde{q}(\mathbf{r})]^{+}} & \text {bare }, \\ {[\tilde{q}(\mathbf{r})+\tilde{l}(\mathbf{r})]^{+}} & \text {screened, } \\ {\left[\sum_{k, \text { p.i. }} \tilde{q}\left(\mathbf{r}_{k}\right)+\tilde{l}\left(\mathbf{r}_{k}\right)\right]^{+}} & \text {screened lattice. }\end{cases}
$$

The screened-lattice version of Eq. (12), where the sum extends over all periodic images of the target particle, 
minimizes the cell-veto rate by merging the periodic images into the primary copy of each particle. The number of target cells $\mathcal{C}_{t}$ is finite, and the target cell of a cell veto can be found extremely efficiently by precomputing the function $Q\left(\mathcal{C}_{a}, \mathcal{C}_{t}\right)$ and employing Walker's alias method or related techniques [20,21] (see Supp. 1). A commented Python implementation of the cell-veto Monte Carlo algorithm using this approach is provided in Supp. 2.

In an alternative version of the cell-veto algorithm, the particle-event rates of Eq. (10) and Eq. (11) are used with explicitly replicated simulation boxes. An infinite number of target cells are considered. The target cell for a cell veto can still be found in constant time by rejection sampling. A vector $\mathbf{r}$ is sampled with probability density $\sim Q(\mathbf{r})$, where $Q$ is an upper bound to the particle-event rate $Q(\mathbf{r}) \geq q(\mathbf{r}+\boldsymbol{\delta})$ for all vectors $\boldsymbol{\delta}$ shorter than the cell diagonal. The target cell $\mathcal{C}_{t}$ is then the cell containing the point $\mathbf{r}_{a}+\mathbf{r}$ (see Supp. 1). The cell-veto rates are somewhat larger than for the lattice-screened version. This may however be offset by the less onerous evaluation of Eq. (10) or Eq. (11) compared to Eq. (12) (surplus particles must be treated with the lattice-screened version).

Both the screened and the screened-lattice particleevent rates overcome the divergence at $n=D-1$ with periodic boundary conditions (see Fig. 3a). Since one cell veto can be handled in $\mathcal{O}(1)$ operations, the computational cost of simulating a fixed timespan is proportional to the rate of cell vetos. For a distance vector $\mathbf{r}=L \mathbf{c}$, the directional derivatives in Eqs (8) and (9) scale as $\sim L^{-n-1}$ and so do the particle-event rates $q$. This implies that, above the point $n=D-1$, the cell-veto rate at constant density scales as $\mathcal{O}\left(L^{D-n-1}\right)=\mathcal{O}\left(N^{1-(n+1) / D}\right)$. For Coulomb forces in $D$ dimensions, $n=D-2$, we find $Q_{\text {tot }} \sim N^{1 / D}$, see Fig. 3b. Thus, in three dimensions, the cell-veto algorithm is of complexity $\mathcal{O}\left(N^{1 / 3}\right)$. This compares favorably with the cost of an $\mathcal{O}\left(N^{3 / 2}\right)$ energy evaluation with Ewald summation in conventional Metropolis Monte Carlo.
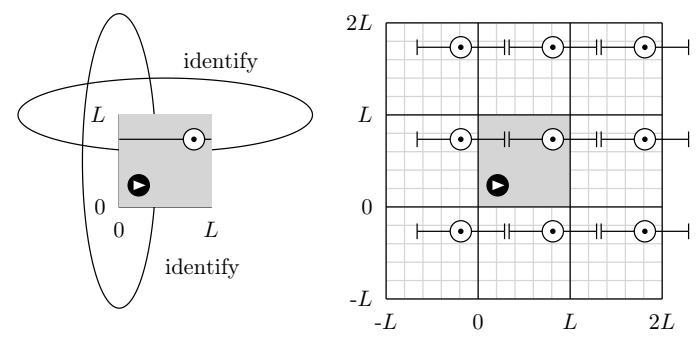

FIG. 4. Periodic particle system with screening line charges. Left: Active and target particles and screening line charge segments in a periodic two-dimensional square box. Right: Folded-out periodic system with image target particles, each of which forms a neutral composite particle together with its screening charge.

In conclusion, we have presented a cell-veto Monte Carlo algorithm that need not compute the system en- ergy. Remarkably, it advances the physical state of the system by one event in $\mathcal{O}(1)$ even for long-ranged interactions. The algorithm introduces none of the cutoffs that come with practical versions of Ewald summation. Strongly long-ranged potentials such as electrostatic forces are handled exactly using screening line charges. The complexity of the algorithm then scales weakly with $N$. It is hoped that the algorithm will permit to access much larger systems than was previously possible. The demo program of Supp. 2, and the $\mathrm{C}++$ version of this algorithm are available online 22 .

* sebastian.kapfer@fau.de

$\dagger$ werner.krauth@ens.fr

[1] N. Metropolis, A. W. Rosenbluth, M. N. Rosenbluth, A. H. Teller, and E. Teller, J. Chem. Phys. 21, 1087 (1953)

[2] W. Krauth, Statistical Mechanics: Algorithms and Computations, Oxford Master Series in Physics (Oxford University Press, UK, 2006).

[3] B. Smit, J. Chem. Phys. 96, 8639 (1992).

[4] P. J. in 't Veld, A. E. Ismail, and G. S. Grest, J. Chem. Phys. 127, 144711 (2007).

[5] S. W. de Leeuw, J. W. Perram, and E. R. Smith, Proc. R. Soc. A 373, 27 (1980)

[6] D. Frenkel and B. Smit, Understanding Molecular Simulation: From Algorithms to Applications, Computational science series (Elsevier Science, 2001).

[7] J. W. Perram, H. G. Petersen, and S. W. de Leeuw, Mol. Phys. 65, 875 (1988).

[8] J. W. Eastwood and R. W. Hockney, J. Comput. Phys. 16, 342 (1974).

[9] L. Greengard and V. Rokhlin, J. Comput. Phys. 73, 325 (1987)

[10] A. Bródka, Chemical Physics Letters 400, 62 (2004).

[11] M. Mazars, Physics Reports 500, 43 (2011).

[12] M. Michel, S. C. Kapfer, and W. Krauth, J. Chem. Phys. 140, $054116(2014)$

[13] E. P. Bernard, W. Krauth, and D. B. Wilson, Phys. Rev. E 80, 056704 (2009)

[14] P. Diaconis, S. Holmes, and R. M. Neal, Annals of Applied Probability 10, 726 (2000).

[15] S. C. Kapfer and W. Krauth, Phys. Rev. Lett. 114, $035702(2015)$

[16] M. Michel, J. Mayer, and W. Krauth, EPL (Europhysics Letters) 112, 20003 (2015)

[17] Y. Nishikawa, M. Michel, W. Krauth, and K. Hukushima, Phys. Rev. E 92, 063306 (2015).

[18] E. A. J. F. Peters and G. de With, Phys. Rev. E 85, 026703 (2012)

[19] We set, for $n \neq 0, \beta U(r)=\beta \varepsilon \times\left((d / r)^{n}-1\right) / n$ and for $n=0, \beta U(r)=\beta \varepsilon \times \ln (d / r)$, i. e., planar Coulomb; here $\varepsilon$ and $d$ are the scales of energy and length. In either case, $\tilde{q}(\mathbf{r})=\beta \varepsilon \times(\hat{\mathbf{e}} \cdot \mathbf{r}) / r^{2+n}$.

[20] A. J. Walker, ACM Trans. Math. Softw. 3, 253 (1977).

[21] G. Marsaglia, W. W. Tsang, and J. Wang, Journal of Statistical Software 11, 1 (2004)

[22] https://github.com/Cell-veto 


\section{Supplementary Item 1: Cell-veto sampling}

The pairwise factorized Metropolis algorithm determines pair events (vetos) and thus avoids to compute the system energy. The cell-veto algorithm takes this strategy one step farther. Instead of scanning all particle pairs for vetos, it first solicits cell vetos (see Fig. 1), which then have to be confirmed on the level of the actual particle positions. Even with periodic boundary conditions, the number of cells remains finite if all the periodic images of a particle are merged into the one located in the primary simulation box (see Eq. $(12)$ ). The next cell veto must be selected from the $N_{\text {cell }}$ cells $\mathcal{C}_{t}$ with a nonzero cell-veto rate. Each cell must be sampled with probability $\sim Q\left(\mathcal{C}_{a}, \mathcal{C}_{t}\right)$, see Fig. S1. This finite discrete-probability sampling problem is best solved through a rejection-free exact algorithm, as Walker's alias method. In Walker's method, the cell-veto rates are reassembled into composite rates consisting of at most two original rates and adding up to exactly the mean cell-veto rate $Q_{\text {mean }}=Q_{\text {tot }} / N_{\text {cell }}$. The cutting-up and reassembling of the $Q\left(\mathcal{C}_{a}, \mathcal{C}_{t}\right)$ constitutes the initialization stage of Walker's method (in the demo program of Supp. 2: in function WalkerSet). In the sampling stage, a cell $\mathcal{C}_{t}$ can be sampled with the proper probability by first sampling the composite rate (as a random integer between 1 and $N_{\text {cell }}$ ) and then deciding between the at most two rates by sampling a uniform random real between 0 and $Q_{\text {mean }}$ (WalkerSample, in the demo program of Supp. 2). This step is constant time and independent of the number of cells.
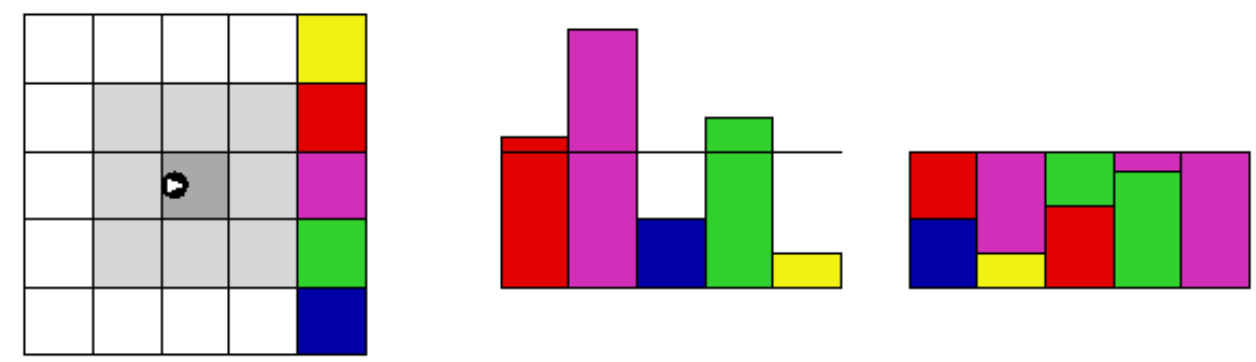

FIG. S1. Cell-veto sampling using Walker's method. Left: The non-nearby cells (5 such cells shown in different colors) may all have finite cell-veto rates. Center: Cell-veto rates in a linear representation. The mean cell rate $Q_{\text {mean }}$ is indicated $(5$ cells shown, again). Right: In the initialization stage of Walker's method, the cell rates are reassembled, at most by pairs, into composite rates.

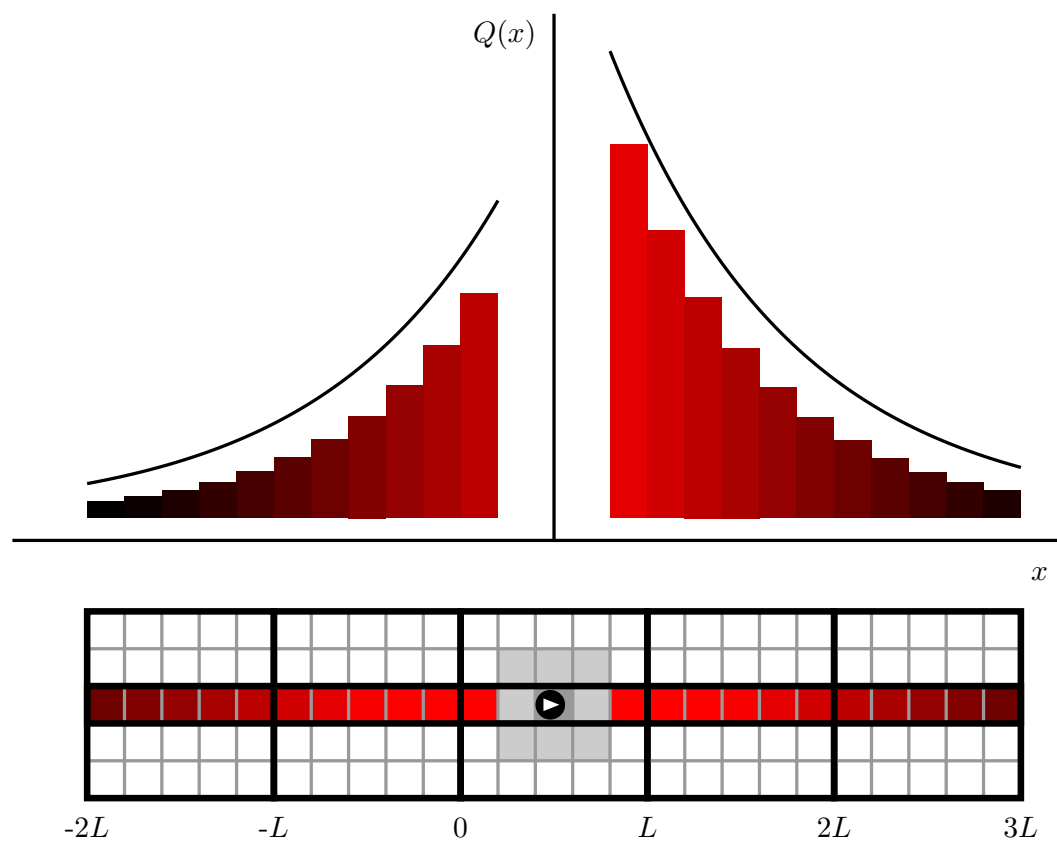

FIG. S2. Cell-veto sampling using rejection sampling with a function $Q(x)$ in a "folded-out" periodic system and an infinite number of cells (one-dimensional representation shown). 
Alternatively, one may also keep the individual cells, that is, work explicitly in the folded-out version of the system, and with the cell-veto rates of Eq. (11) that consider each periodic copy of a target cell individually. The number of cells is now countably infinite. Nevertheless, it is easy to devise a rejection-sampling strategy using a function that is easy to sample, integrable to infinity, and an upper bound to the cell-veto rate (see Fig. S2 for a one-dimensional representation). A point $x$ sampled from the probability distribution $\sim Q(x)$ identifies a cell. If that cell contains a target particle $t$, a particle event is triggered with probability $q\left(\mathbf{r}_{t}\right) / V_{\text {cell }} / Q(x)$. In the folded-out formulation of the cell-veto algorithm, surplus particles must still be merged with their periodic images, in order to keep their number finite.

\section{Supplementary Item 2: Demo implementation of the cell-veto algorithm}

The demo implementation of the cell-veto Monte Carlo algorithm, the program demo_cell.py, is written in the Python 2 programming language. $N$ particles are simulated in a two-dimensional square box of length 1 with periodic boundary conditions, and with an $1 / r$ pair potential that is periodically continued. A regular square grid with $L^{2}$ cells is superimposed to the system. Cells are numbered from 0 to $L^{2}-1$. The screened-lattice particle-event rate of Eq. (12) is implemented (naively). Walker's method is used for sampling the veto cells.

In the setup stage of demo_cell.py, particles are initialized to random positions, and the cell-veto rates are computed between the active cell $\mathcal{C}_{a}=0$ and all other target cells that are not nearby $\mathcal{C}_{a}=0$. The function translated_cell transfers this calculation (with $\mathcal{C}_{a}=0$ ) to arbitrary cell pairs $\left(\mathcal{C}_{a}, \mathcal{C}_{t}\right)$. Specifically, the cell-veto rate is defined as the maximum of the particle-event rate over all positions, as indicated in Eq. (5). For this demo program, it is assumed that the maximum particle-event rate is attained for $\mathbf{x}_{a}$ and $\mathbf{x}_{t}$ on the boundary of $\mathcal{C}_{a}$ and $\mathcal{C}_{t}$, respectively, and discrete points in the list cell_boundary are used. For the demo version, the lattice-screened particle-event rate of Eq. (12) is determined by a naive direct summation of the images of the target particle and its screening line charge (see function pair_event_rate), rather than by an efficient function evaluation. The initializaton of Walker's alias method, as explained in Supp. 1, concludes the setup stage of demo_cell.py.

In one iteration of the sampling stage of demo_cell.py, particles advance by a total distance chain_ell (see [12, 13]) in a fixed direction. This direction of motion is first sampled (from $+x$ or $+y$ ). In the demo version, only the $+x$ move is implemented explicitly $(+y$ moves are implemented indirectly by flipping all particle coordinates $\left.\left(x_{i}, y_{i}\right) \rightarrow\left(y_{i}, x_{i}\right)\right)$. At the beginning of this iteration (given that such a flip may have taken place) particles are reclassified into target particles associated to cells (at most one per cell), and surplus particles. (Each cell must contain at most one particle, in order for the cell-veto rate to be an upper limit for the particle-event rate from all particles within the cell). The active particle is then sampled uniformly among all particles in the system. At each step of the iteration, the step size delta_s to the next cell veto is sampled from the total cell-veto rate $Q_{\text {tot }}$. The cell veto may be preempted by the end of the chain, after displacement chain_ell. It is also checked whether the cell veto occurs after the active particle crosses the cell limit: We must trigger an event when the cell boundary is reached, as the set of nearby particles then changes. If the cell veto is indeed confirmed on the particle level, it is put into competition with events triggered by nearby or surplus particles. In the demo version, the particle-event rates for nearby or surplus particles are computed in a simplified way.

The demo_cell.py program (see below) was tested against a straightforward implementation of the Metropolis algorithm, and against the $\mathrm{C}++$ version (see https://www.github.com/cell-veto/postlhc/).

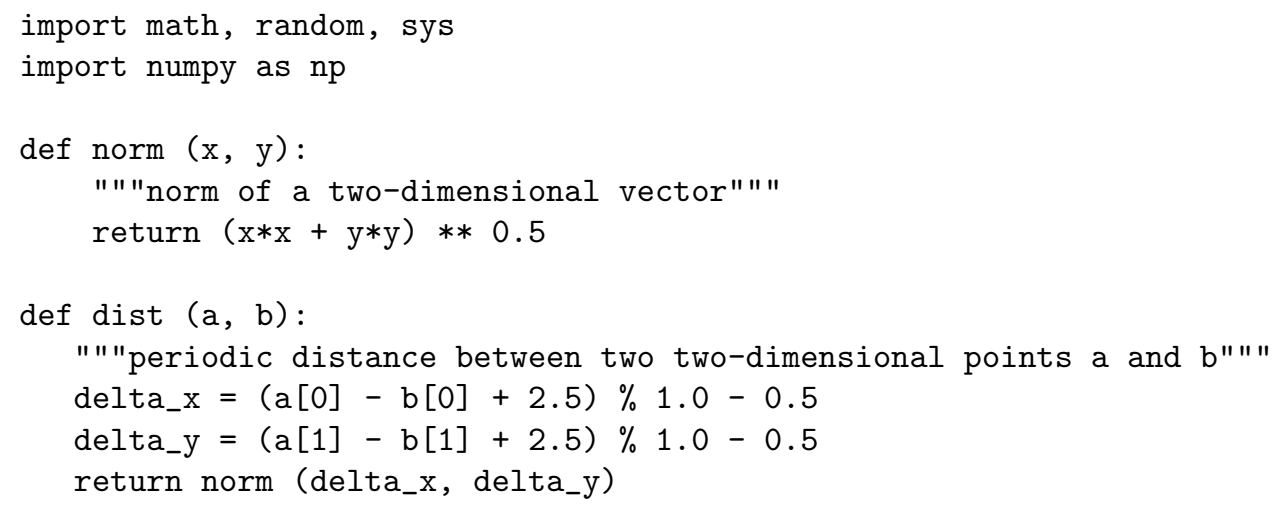




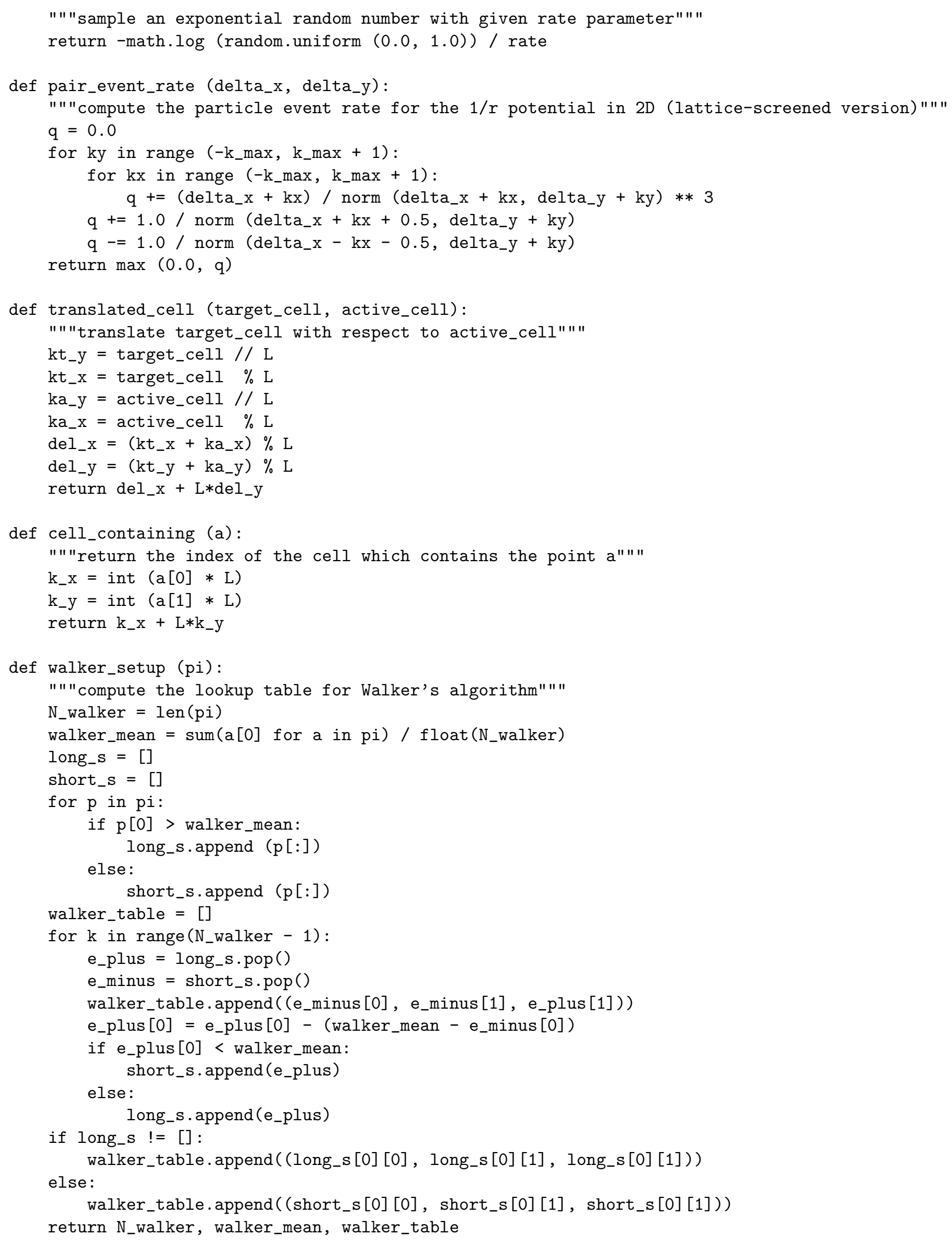




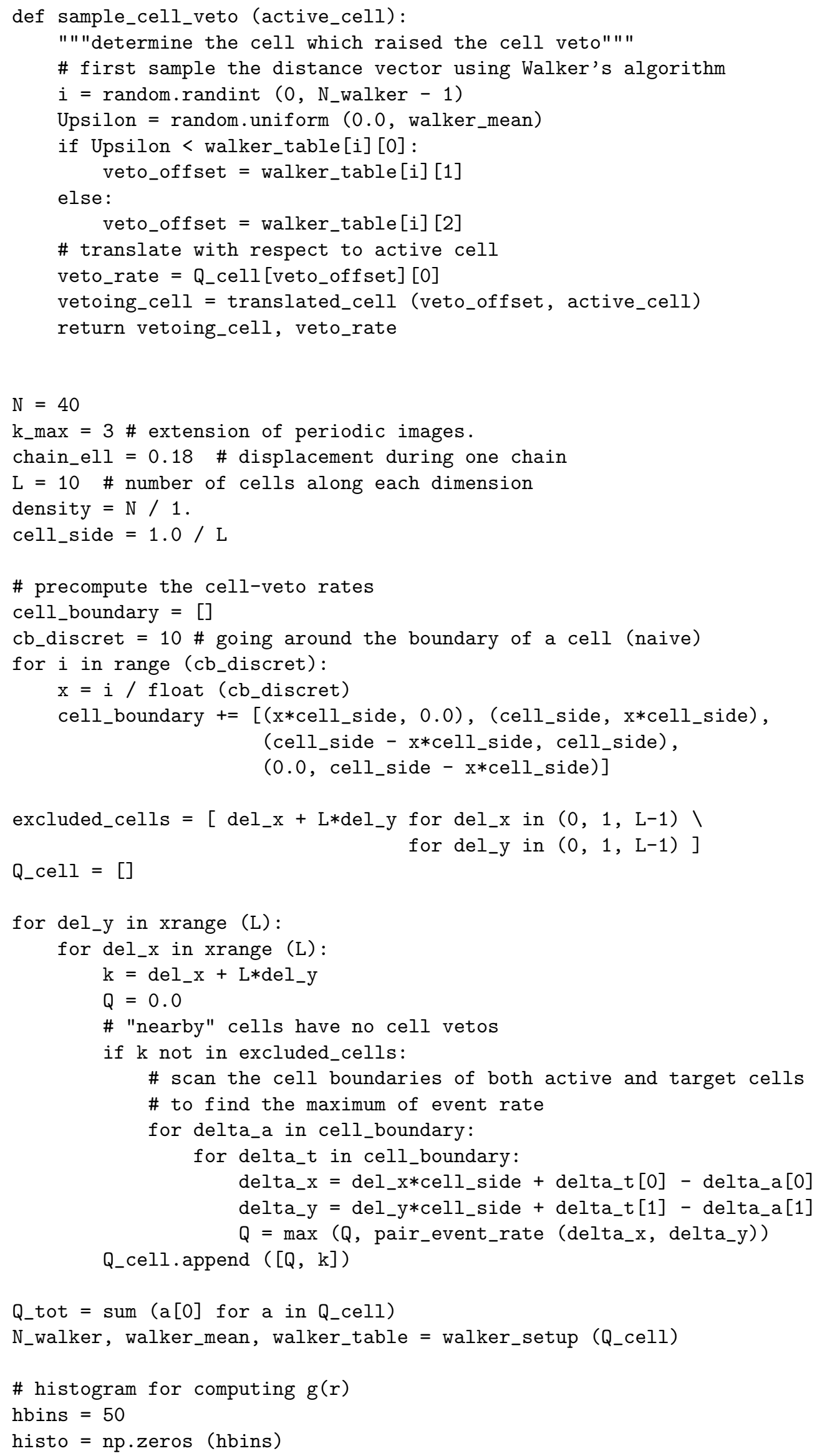




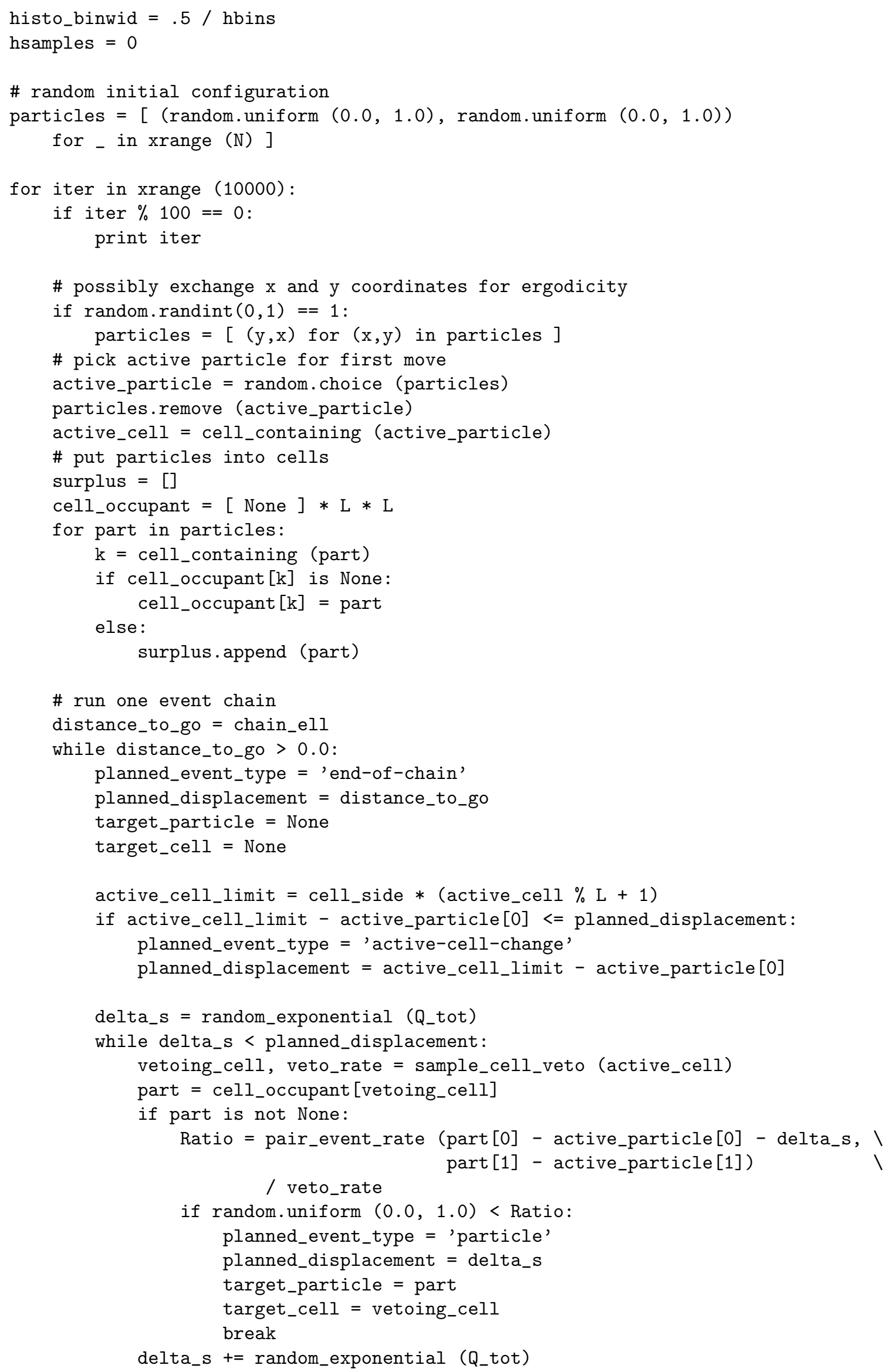




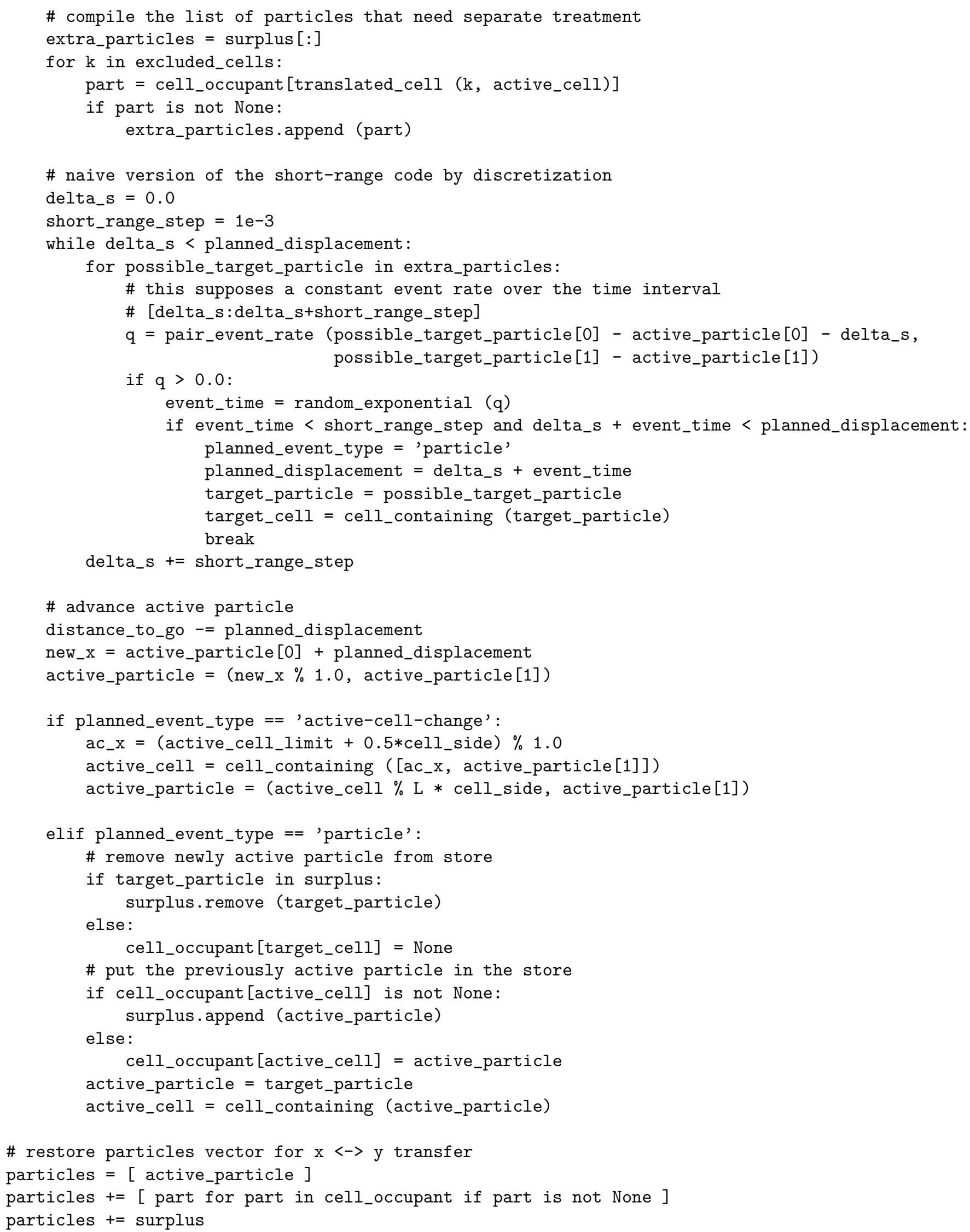




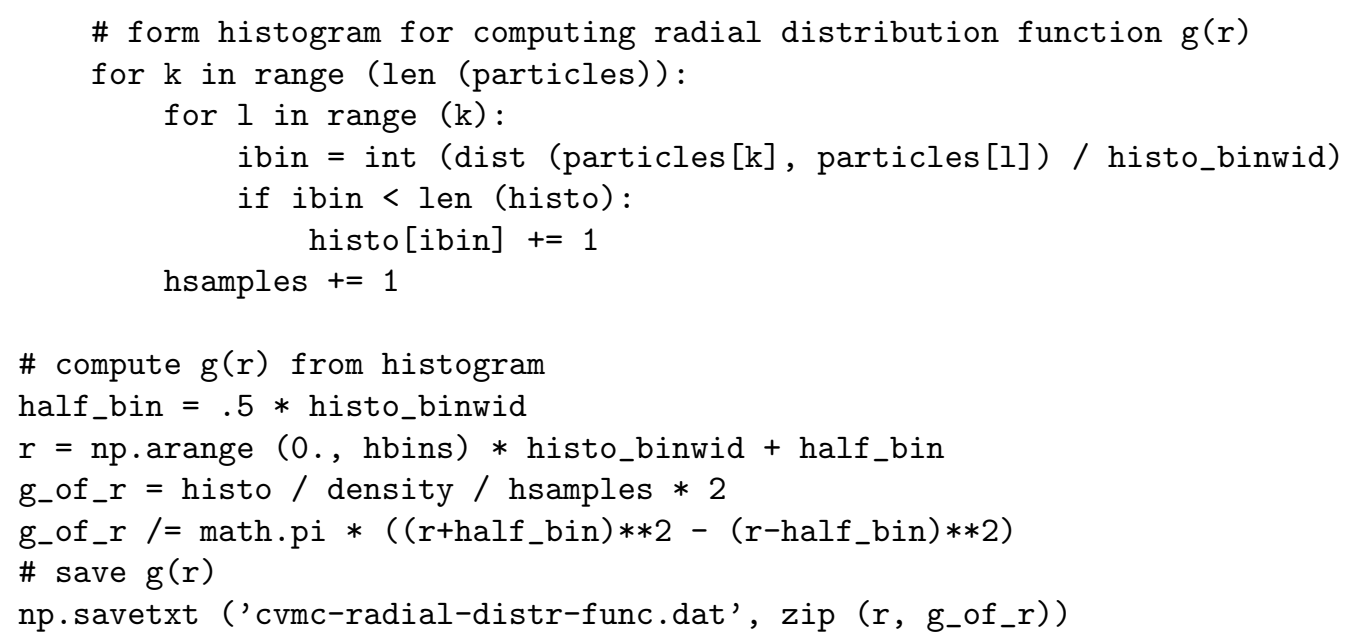

